\title{
Educação
}

\section{Novas estratégias e velhos erros: Uma Introdução ao Cálculo para Alunos de Engenharias}

\author{
Jorge Carvalho Brandão ${ }^{1}$; Elisângela Bezerra Magalhães ${ }^{2}$; Emília da Costa ${ }^{3}$
}

\begin{abstract}
Resumo: Este trabalho relata a experiência de inverter tópicos do Cálculo Diferencial e Integral com uma variável visando responder um questionamento: por qual motivo seguir sequência didática de livros adotados? Método dos trapézios para cálculo de uma área acima do eixo dos x e entre parábolas côncavas para baixo foi um ponto de partida, bem como algumas aplicações das integrais, como trabalho e centro de massa, usando o referido método. Não obstante algumas inversões didáticas, a razão de certas expressões, como o limite fundamental da trigonometria, eram apresentadas a partir de uma contextualização. Embora o uso de novas estratégias focando uma aprendizagem mais significativa, constatou-se que os mesmos erros no tradicional ensino de limites, derivadas e integrais foram observados.
\end{abstract}

Palavras-Chaves: Erros. Estratégias. Cálculo.

\section{New strategies and old mistakes: An Introduction to Calculus for Engineering Students}

\begin{abstract}
This paper reports the experience of reverse topics Differential and Integral Calculus with a variable aiming to answer one question: For which reasons to follow the didactic sequence of the adopted books? The method of the trapezes to calculate the area above the $\mathrm{x}$ axis between concave down parabolas has a starting point as well as applications of integrals, such as work and center of mass using the above method. Despite some didactic inversions, why certain expressions, as the fundamental limit of trigonometry, were submitted from contextualization. Although the use of new strategies focusing on a more meaningful learning, it was found that the same mistakes in the traditional teaching of limits, derivatives and integrals were observed.
\end{abstract}

Keywords: Errors. Strategies. Calculation.

\section{Introdução}

Vários livros didáticos de Cálculo Diferencial e Integral com uma variável, como Stewart (2010) e Thomas (2009) entre outros, seguem a seguinte sequência didática: Introdução ao Cálculo (ou revisão de funções), Limites; Derivadas (regras e aplicações) e Integrais (indefinidas, definidas, regras e aplicações). Por qual motivo?

Poucos pesquisadores respondem de maneira satisfatória o questionamento anterior. A resposta mais frequentemente indicada é o amadurecimento dos conteúdos.

\footnotetext{
${ }^{1}$ Doutor em Educação. Professor Matemática para Engenharias da Universidade Federal do Ceará. e-mail: profbrandao@ufc.br

${ }^{2}$ Doutoranda em Educação Brasileira Universidade Federal do Ceará - Mestre em Educação pela UFC. Psicopedagoga Clínica. e-mail: lala2magalhaes@gmail.com

${ }^{3}$ Mestranda em Educação Brasileira pela Universidade Federal do Ceará. Química e-mail: emilialimaufcg@hotmail.com
} 
Com efeito, algumas aplicações das integrais incluem o comprimento de arco que envolve derivação. Vale ressaltar que muitas integrais são concebidas como anti-derivadas. Uma derivada é um tipo particular de limite. Assim, segue-se a sequência.

Por sua vez, observa-se na resolução de provas de alguns discentes que podem errar questões de derivação e, no entanto, acertar de maneira consciente questões de integração. É por causa do dito amadurecimento ou é mero acaso? Assim sendo, este trabalho objetiva analisar, mesmo que de maneira sucinta, se a modificação na forma de apresentação de conteúdos na disciplina de Cálculo Diferencial e Integral com uma variável implica em uma não aprendizagem dos mesmos.

Como uma "motivação" para muitos discentes estudarem são avaliações, tratamos da avaliação no próximo tópico. Com efeito, precisamos de um norte em relação à metodologia utilizada. Os autores são "antigos" porque não encontramos, de maneira satisfatória, nos novos autores novas formas de perceber a avaliação.

\section{Avaliação}

Por que avaliar? Conforme Hoffmann (1994) a avaliação tem como objetivo favorecer ações educativas as quais possibilitem novas descobertas. A avaliação destina-se à melhoria do ciclo de vida. Por conseguinte, conforme Luckesi (1994), a avaliação deve ter um caráter diagnóstico, criando bases para tomadas de decisões na perspectiva de maior satisfatoriedade nos resultados.

Tanto Luckesi (1994) quanto Hoffmann (1994) salientam a avaliação como um instrumento subsidiário da prática educativa. Assim sendo, para que haja uma avaliação da aprendizagem satisfatória é preciso coletar, analisar e sintetizar as condutas dos educandos frente à determinada atividade dirigida pelo professor.

Neste trabalho as atividades dirigidas são confecções de situações-problema em oficinas realizadas com educadores e graduandos. Jogos com tangram servem de referencial para construções geométricas.

A avaliação informa ao professor o que foi aprendido pelo estudante. Longe de ser apenas final do processo de ensino, a avaliação se inicia quando os estudantes põem em jogo seus conhecimentos prévios e continua a se evidenciar durante toda situação escolar (BRASIL, 1998).

\section{Perguntas que norteiam a avaliação da aprendizagem}


Há quatro perguntas que devem nortear uma boa avaliação da aprendizagem, aconteça ela no ensino fundamental, médio ou superior (BARBOSA, 2006).

\section{$1^{a}$ - Quem avalia?}

$\mathrm{Na}$ escola tradicional os papéis ficavam bem definidos: cabia ao professor a autoridade suprema da avaliação, cujos resultados eram inquestionáveis, e o aluno era o "objeto" da avaliação.

Perrenoud (1999) afirma que a avaliação fica em conflito entre duas lógicas, diametralmente opostas: normativa e formativa. A avaliação normativa, colocada a serviço da seleção, apenas cria hierarquias de excelência, pois "os alunos são comparados e depois classificados em virtude de uma norma de excelência, definida no absoluto ou encarnada pelo professor e pelos melhores alunos". (PERRENOUD, 1999, p. 11)

A avaliação formativa, colocada a serviço das aprendizagens, torna-se mais uma estratégia pedagógica de luta contra o fracasso e as desigualdades. Inclui o cuidadoso conhecimento da aprendizagem dos alunos e a aplicação de estratégias diferenciadas para cada grupo.

Não se trata, no entanto, de descuidar alguns aspectos formais da avaliação, que precisam ser cumpridos "[...] a avaliação formativa não dispensa os professores de dar notas ou de redigir apreciações, cuja função é informar os pais ou a administração escolar sobre as aquisições dos alunos, fundamentando a seguir decisões de seleção ou de orientação". (PERRENOUD, 1999, p. 16).

Existem, portanto, três formas de avaliação, que não retiram do professor a responsabilidade de realizá-la, mas que a tornam compartilhada, mais democrática e mais aproximada da segunda modalidade de que fala Perrenoud. Dependendo da modalidade, varia a resposta à primeira pergunta "Quem avalia?". São elas (BARBOSA, 2006):

- Hetero-avaliação: consiste na valorização do rendimento escolar feita por pessoas distintas do próprio aluno, fundamentalmente o professor.

- Auto-avaliação: expressa o juízo de valor que o aluno faz do rendimento educativo que teve.

- Avaliação mista ou co-avaliação: tem lugar quando o docente e o aluno avaliam em conjunto as atividades ou o rendimento deste; quando ambos analisam determinadas tarefas ou rendimentos. Pode incluir, como agentes avaliadores, outros alunos, no caso de atividades realizadas em grupo.

Sempre que o professor abre mão de monopolizar o poder da avaliação, compartilhando-o com outras pessoas, o processo se torna mais democrático e o comprometimento e a participação de todos, ao avaliarem e serem avaliados, crescem consideravelmente.

\section{$2^{a}$ - Quando avaliar?}

A resposta é imediata e única: sempre. 
Se considerarmos a aprendizagem como um processo contínuo, global e cumulativo, as avaliações pontuais sempre incorrem em sérios riscos de erro. A avaliação deve ser, portanto, contínua.

Isto fica mais claro, ao ser feito destaque das quatro modalidades de avaliação do rendimento acadêmico dos alunos:

- Diagnóstica - de início de processo, permite o conhecimento inicial do aluno, dos conceitos que já possui, do seu estilo de aprendizagem.

- Formativa - acompanha o processo, permitindo as correções e ajustes necessários.

- Somativa - a única realizada no modelo tradicional de Educação, que permite a classificação dos alunos de acordo com padrões estabelecidos previamente.

- Meta-avaliação - tem como foco o próprio sistema de avaliação utilizado, sua validade e fidedignidade.

Avaliação contínua refere-se a este ciclo de avaliações que se articulam permitindo, ao fim, regular e corrigir as falhas do próprio sistema de avaliação utilizado.

Destaca-se, neste processo de continuidade da avaliação, a modalidade formativa. Realizada durante o processo de aprendizagem, torna-se mais um instrumento para aprimorar sua realização.

\section{$3^{a}$ - Como avaliar?}

Quanto às estratégias avaliativas, podemos propor um saudável "vale-tudo" metodológico. Tudo o que o aluno produz pode demonstrar a aprendizagem realizada e, consequentemente, ser avaliado.

Considerando-se as situações formais de avaliação, planejadas pelo professor (provas, testes, atividades individuais ou grupais) e as situações informais, ocorridas no cotidiano da sala de aula, e que também se prestam para esta finalidade (principalmente quanto à avaliação formativa).

É conveniente lembrar, ainda, que as expressões em variadas linguagens devem ser consideradas para fins avaliativos, complementando aquelas que são propostas por escrito, tão comuns em nossa realidade (BARBOSA, 2006).

\section{$4^{a}$ - Para que avalia?}

Busca-se:

- Verificar o ritmo e os estilos de aprendizagem dos alunos.

- Realimentar o processo ensino-aprendizagem, permitindo efetuar correções. 
- Identificar os conteúdos e objetivos mais importantes.

- Detectar e corrigir os erros mais freqüentes.

- Comparar o desempenho demonstrado pelos alunos, com os objetivos inicialmente definidos.

- Verificar a possibilidade dos alunos atingirem estágios posteriores de aprendizagem.

- Avaliar o próprio sistema de avaliação utilizado.

- Verificar a adequação das atividades e estratégias.

Feitas tais colocações em relação a avaliação, não podemos esquecer dos discentes que possuem dificuldades de aprendizagem neste campo do saber

\section{A Experiência Realizada e Atividades Propostas}

A forma de investigação foi a resolução escrita de avaliações realizadas durante um ano letivo. Com efeito, a disciplina que sofreu a modificação é anual na Universidade Federal do Ceará. É conhecida como Cálculo Fundamental e tem carga horária de 128 h/aula. Os encontros eram realizados duas vezes por semana com duas horas aula cada um.

A turma observada, em relação à mudança da sequência didática, durante o ano de 2012 foi de alunos da Engenharia Química, com um total de 82 discentes matriculados. Não obstante os encontros em sala de aula, foi criada uma página no face book para uma maior interação entre os estudantes. Alguns discentes também recorriam a aulas de exercícios com monitores em horários extras à sala de aula.

Mantivemos em outras duas turmas de Cálculo Fundamental (uma de Engenharia Mecânica com 27 alunos e outra de Engenharia Metalúrgica com 62 alunos) a sequência didática apresentada nos livros de Cálculo do Stewart (2009) e do Thomas (2010), respectivamente, para engenharias Mecânica e Metalúrgica. As avaliações escritas nessas turmas eram as mesmas ${ }^{1}$.

É claro que, diante da mudança da sequência didática na turma de Engenharia Química, a "essência" de algumas questões eram replicadas. Isto é, aplicações de derivadas e integrais impróprias eram exigidas em provas, só não eram as mesmas questões.

Sendo avaliações escritas, conforme Cury (2007), tornou-se necessária uma visão geral de cada prova para caracterizar os tipos de soluções em totalmente corretas, parcialmente corretas ou incorretas. Como pequeno diferencial nesse trabalho, além de analisar cada prova individualmente, comparava-a com as demais provas do grupo.

\footnotetext{
${ }^{1}$ As avaliações eram realizadas nos mesmos dias e horários, para evitar "vazamento" de informações.
} 
Com efeito, segundo McDonald (2003), cometem-se menos erros em correções de provas se todas as provas forem corrigidas em sequência de questões. Isto é, corrigir a primeira questão de todas as provas, depois a segunda questão e assim sucessivamente. Em seus estudos, ele observou variação de até um ponto por questão, em uma escala de zero a dez, quando as correções eram comparadas.

Entendendo: um professor aplica uma prova. Corrige todas as questões da prova do aluno 01, depois todas do aluno 02 e assim sucessivamente. Todavia, o docente não registra as correções da prova na prova, faz apenas anotações em um gabarito à parte. Após 08 dias, o mesmo docente vai corrigir as mesmas provas, sendo questão por questão conforme parágrafo anterior. Após correção, compara com gabarito.

Assim sendo, as correções das avaliações com a turma da Engenharia Química seguiram a ideia proposta por McDonald (2003). Não obstante, as resoluções tiveram, a partir das propostas de Cury (2007), até cinco caracterizações: Completamente satisfatórias (mais de 96\% da questão); Bem satisfatórias (de $76 \%$ a $95 \%$ da questão); Satisfatórias (de $51 \%$ a $75 \%$ da questão); Parcialmente satisfatórias (de $26 \%$ a $50 \%$ da questão) e Insatisfatórias (menos de $25 \%$ da questão).

Como saber a porcentagem de uma questão? A partir das etapas ou partes que compõe uma resolução correta de cada questão. Repare no uso do artigo indefinido uma. Por conseguinte, uma mesma questão pode ter para alunos distintos a caracterização Parcialmente satisfatória ou Completamente satisfatória dependendo da ideia apresentada em dada resolução. No próximo tópico serão apresentados alguns exemplos.

Todavia, por se tratar de um relato, os resultados obtidos com a turma de observação das estratégias serão comparados com resultados de outras turmas de mesmo curso e mesma disciplina nos moldes tradicionais. Vale ressaltar: resultados atrelados aos tipos de erros e não às notas médias obtidas pelas turmas.

\section{Percurso Metodológico}

Como já mencionado, as avaliações eram diferentes, todavia mantinham o mesmo grau de dificuldade e cobrança. Exemplificando: enquanto a primeira prova das turmas de Engenharia Mecânica e Metalúrgica contemplou limites e regras de derivação, com aplicações, tais conteúdos só foram abordados na segunda avaliação da Engenharia Química. Só a quarta avaliação e a avaliação final $^{3}$ foram iguais para todos.

\footnotetext{
${ }^{2}$ Aplicação de integrais impróprias e coordenadas polares

${ }^{3}$ Que englobou todo o assunto visto no ano. A avaliação final foi aplicada no mesmo dia e horário para todos os discentes que não conseguiram aprovação direta.
}

35 Id en line Revista de Psicologia. Ano 9, No. 28. Novembro/2015 - ISSN 1981-1179. Edição eletrônica em http://idonline.emnuvens.com.br/id 
Em relação à mudança na sequência didática, as primeiras aulas contemplaram aplicações de integrais definidas sem, no entanto, especificar que os problemas envolviam tais conteúdos. A ideia inicial foi calcular a área compreendida entre os eixos $\mathrm{x}$ e y e a curva $\mathrm{y}=9-\mathrm{x}^{2}$. O método dos trapézios foi utilizado.

Em seguida utilizou-se a ideia de dividir em partes iguais o intervalo [0,3] (aqui há uma sequencia do exemplo apresentado no parágrafo anterior, em sala, vários outros exemplos foram refeitos). Sempre questionando o corpo discente o que ocorria com o aumento na quantidade de intervalos e consequente tamanho de cada um. Cálculo de trabalho e centro de massa também foram inseridos, com resolução aproximada.

Foi preparada a noção de limite. $\mathrm{O} \varepsilon$ e o $\delta$ foram apresentados focando também o significado de cada letra, respectivamente, erro cometido (no eixo dos y) diante de um desvio na variação no eixo dos $\mathrm{x}$. Vale ressaltar que pequenas atividades concretas, tais como um discente vendado e usando uma bengala longa percorrendo um dado percurso para tentar vivenciar $\varepsilon$ e $\delta$.

Fornecida a ideia formal (definição de limites com respectivas operações), novamente foram utilizadas aplicações. Por exemplo, se no movimento harmônico simples (logo, há movimentos que não são harmônicos ou não são simples, argumentaram alguns discentes com base nas palavras utilizadas $), \mathrm{v}(\mathrm{t})=\operatorname{sen}(\mathrm{t})$ representa a velocidade, então para uma pequena variação do tempo caímos no limite fundamental da trigonometria.

Com efeito, $\lim _{t \rightarrow 0}[v(t)-v(0)] /[t-0]=\lim _{t \rightarrow 0}[\operatorname{sent}-\operatorname{sen} 0] / t=\lim _{t \rightarrow 0}(\operatorname{sen} t / t)$. Ou seja, dentro de um contexto são apresentados os limites mais utilizados. Vale ressaltar que as palavras fazem sentido. Isto é, é limite fundamental da trigonometria porque os demais limites que envolvem funções trigonométricas e resultam em 0/0 são resolvidos direta ou indiretamente por ele (comparar com a relação fundamental da trigonometria).

Após o conteúdo regras de derivação, onde cada regra era argumentada e debatida de maneira formal. Em seguida, foram apresentadas as regras de L'Hopital. Observou-se que muitos discentes faziam uso indiscriminadamente.

Como só a quarta avaliação e a avaliação final foram iguais, as questões com as respectivas categorias de análise dos erros da avaliação final, feita por 84 discentes, sendo 36 da Engenharia Química, 18 da Engenharia Mecânica e 30 da Engenharia Metalúrgica. A descrição de cada uma delas é uma forma de interpretar a atuação do discente na resolução da questão, tentando compreender os possíveis motivos dos erros. Não será abordada a pontuação de cada questão.

Primeira questão (52 acertaram a questão, sendo 23 da Engenharia Química, 11 da Engenharia Mecânica e 16 da Engenharia Metalúrgica):

Texto de Apoio: Uma maneira de calcular aproximadamente a área de uma região compreendida abaixo da função contínua $y=f(x)$, acima do eixo dos $x$ e limitada lateralmente pelas retas $\mathrm{x}=\mathrm{a}$ e $\mathrm{x}=\mathrm{b}$ é dividir o intervalo $[\mathrm{a}, \mathrm{b}]$ em $\mathrm{n}$ partes iguais e confeccionar trapézios. Sendo $\Delta \mathrm{x}=$ 
$(b-a) / n, a=x_{0}, b=x_{n}$ então, sabemos que pelo referido método, a área é cerca de: $(\Delta x / 2) *\left\{f\left(x_{0}\right)+\right.$ 2. $\left.\left[\mathrm{f}\left(\mathrm{x}_{1}\right)+\ldots+\mathrm{f}\left(\mathrm{x}_{\mathrm{n}-1}\right)\right]+\mathrm{f}\left(\mathrm{x}_{\mathrm{n}}\right)\right\}$.

Aplicação: Qual a área aproximada da região limitada no primeiro quadrante abaixo da curva $\mathrm{y}=4-\mathrm{x}^{2}$. Use $\mathrm{n}=5$.

Principais erros observados:

a) O conteúdo do texto de apoio foi abordado durante duas aulas consecutivas. Foi apresentado na prova com o intuito de auxiliar discentes. Todavia, alguns discentes não fizeram a questão argumentando não entender o enunciado. Com efeito, em prova não é permitida consulta ao docente, sendo indicado aos discentes que ler e interpretar cada questão faz parte da avaliação. Erro de compreensão do enunciado. 21 alunos não a fizeram.

b) Uso indevido da expressão. Não identificaram os $x_{i}$. Com efeito, pelo contexto discentes deveriam obter $\mathrm{x}_{1}=\mathrm{x}_{0}+\Delta \mathrm{x}, \mathrm{x}_{2}=\mathrm{x}_{1}+\Delta \mathrm{x}, \mathrm{x}_{3}=\mathrm{x}_{2}+\Delta \mathrm{x}$, reorganizando, deveriam chegar $e m \mathrm{x}_{\mathrm{i}}=\mathrm{x}_{0}+\mathrm{i} . \Delta \mathrm{x}$. Fizeram o cálculo como sendo um único trapézio: $[\mathrm{f}(0)+\mathrm{f}(2)]^{* 5 / 2}$. Erro consiste em forçar a fórmula, isto é, por causa da palavra trapézio, desconsideraram fórmula dada, tentando ganhar alguma pontuação da questão. Oito alunos seguiram essa linha de raciocínio.

c) Uso indevido da expressão. Alguns identificaram os $\mathrm{x}_{\mathrm{i}}$. Com efeito, pelo contexto chegaram em $\mathrm{x}_{\mathrm{i}}=\mathrm{x}_{0}+\mathrm{i} . \Delta \mathrm{x}$. Erro: somaram os $\mathrm{f}\left(\mathrm{x}_{\mathrm{i}}\right)$, esquecendo que os intermediários são multiplicados por dois. Outros de maneira coerente chagaram em $\left\{\mathrm{f}\left(\mathrm{x}_{0}\right)+2 \cdot\left[\mathrm{f}\left(\mathrm{x}_{1}\right)+\ldots+\right.\right.$ $\left.\left.\mathrm{f}\left(\mathrm{x}_{\mathrm{n}-1}\right)\right]+\mathrm{f}\left(\mathrm{x}_{\mathrm{n}}\right)\right\}$. Esqueceram de multiplicar por $(\Delta \mathrm{x} / 2)$. Erro menos grotesco. Falta de atenção! Três discentes se enquadram nessa observação.

Segunda questão (33 acertaram a questão, sendo 12 da Engenharia Química, cinco da Engenharia Mecânica e 16 da Engenharia Metalúrgica):

Texto de Apoio: Quando uma partícula está se movendo com uma função deslocamento s(x) a velocidade instantânea é dada por $\lim _{\mathrm{h} \rightarrow 0}[\mathrm{~s}(\mathrm{x}+\mathrm{h})-\mathrm{s}(\mathrm{x})] / \mathrm{h}$.

Aplicação: A função $\mathrm{s}(\mathrm{x})=\cos (2 \pi \mathrm{x}+\pi / 3)$ representa o deslocamento de uma partícula. Qual sua velocidade instantânea?

Principais erros observados:

a) O conteúdo do texto de apoio foi abordado durante as aulas. Foi apresentado na prova com o intuito de auxiliar discentes. Todavia, alguns discentes não fizeram a questão argumentando não entender o enunciado. Com efeito, em prova não é permitida consulta ao docente, sendo indicado aos discentes que ler e interpretar cada questão faz parte da avaliação. Erro de compreensão do enunciado. 22 alunos não a fizeram. 
Vale ressaltar que há discentes que não resolveram a questão anterior e aqui se enquadram.

b) Aplicação da regra de L'Hopital, pois argumentaram ser um limite com a forma indeterminada $0 / 0$. Todavia, não derivaram coerentemente a função $\mathrm{s}(\mathrm{x})$ - esqueceram da regra da função composta. Erro na aplicação das regras de derivação. 17 discentes cometeram o referido erro.

c) Desenvolveram a função, usando o cosseno da soma: $\cos (2 \pi x+\pi / 3)=$ $\cos (2 \pi x) \cdot \cos (\pi / 3)-\operatorname{sen}(2 \pi x) \cdot \operatorname{sen}(\pi / 3)$, substituíram os valores dos seno e do cosseno de $\pi / 3$. Todavia, após usarem o fato de que o limite de uma soma é a soma dos limites, não concluíram os cálculos. Erro consiste em não saber usar as consequências do limite fundamental da trigonometria. 12 discentes direta ou indiretamente se enquadram nesse tipo de erro.

Terceira questão (36 acertaram a questão, sendo 17 da Engenharia Química, oito da Engenharia Mecânica e 11 da Engenharia Metalúrgica):

Texto de Apoio: Dizemos grosso modo que uma função contínua em um intervalo (c, d) tem um mínimo local em $\mathrm{m}$ se sua derivada f' é negativa em (c, m) e positiva em (m, d). Por sua vez, se sua derivada f' é positiva em (c, m) e negativa em (m, d), então tem um máximo local. Sabemos que, pelo teste da derivada segunda, se $\mathrm{f}^{\prime}(\mathrm{m})=0$ e $\mathrm{f}^{\prime}(\mathrm{m})$ existe, e é diferente de zero, então teremos mínimo local em $\mathrm{m}$ se f' $(\mathrm{m})>0$ e máximo local em $\mathrm{m}$ se f' $(\mathrm{m})<0$.

Aplicação: Dentre todos os triângulos inscritos em uma semicircunferência, qual o de menor área?

Principais erros observados:

a) O conteúdo do texto de apoio foi abordado durante as aulas. Foi apresentado, inclusive, problema parecido: retângulo inscrito em semicircunferência. Alguns discentes resolveram o problema considerando retângulo em vez de triângulo. Todavia, erraram ou na confecção da função ou na derivação. Erro de interpretação do enunciado. 12 discentes observados.

b) Erro associado à confecção da função área. Não usaram o fato de um triângulo inscrito em uma semicircunferência ser retângulo. Fizeram como um triângulo sendo isósceles. Erro relacionado com a falta de conhecimentos prévios, no caso, triângulo inscrito em uma semicircunferência ser retângulo. 13 discentes o cometeram.

c) Usaram o fato de um triângulo inscrito em uma semicircunferência ser retângulo. Por sua vez, erraram na derivada da função área. Erro associado às regras de derivação. Nove alunos cometeram. 
d) Fizeram tudo coerentemente, exceto verificar que o valor encontrado minimiza a área. Erro cometido por 14 discentes.

Quarta questão (53 acertaram a questão, sendo 24 da Engenharia Química, 12 da Engenharia Mecânica e 17 da Engenharia Metalúrgica):

Texto de Apoio: Seja C uma constante. Sabemos que $\int \cos x d x=\operatorname{sen} x+C$ porque ao derivarmos sen $x+C$ obtemos o cosx. Também sabemos que $\int \mathrm{d} x /\left(1+\mathrm{x}^{2}\right)=\operatorname{arctg} \mathrm{x}+\mathrm{C}$ pelo mesmo motivo. Quando há uma função composta, fazemos uma mudança de variável. Por exemplo, $\int \mathrm{e}^{\mathrm{ax}} \mathrm{dx}=$ $\mathrm{e}^{\mathrm{ax}} / \mathrm{a}+\mathrm{C}$, com $\mathrm{a} \neq 0$. Idem para expressão $\int(a x+b)^{\mathrm{n}} \mathrm{dx}=(\mathrm{ax}+\mathrm{b})^{\mathrm{n}+1} / \mathrm{a} \cdot(\mathrm{n}+1)+C$, se $\mathrm{n} \neq-1$ e $\int \mathrm{dx} /(\mathrm{ax}+$ b) $=(1 / a) \cdot \ln |a x+b|+C$.

Aplicação: Uma partícula tem uma velocidade $\mathrm{v}(\mathrm{x})=-\pi \operatorname{sen}(\pi \mathrm{x}+\pi / 4)$. Qual a função deslocamento? Dado: $\mathrm{s}(1 / 4)=0$.

Principais erros observados:

a) Má compreensão do enunciado ocasionando a não resolução do problema. Erro cometido por seis discentes.

b) Erro associado a integração sem considerar que há composição na função. Erro de integração. 16 discentes cometeram.

c) Integraram coerentemente mas não fizeram uso da informação $s(1 / 4)=0$. Todos desconsideraram a constante. Erro de compreensão do que seja uma integral indefinida. Nove discentes se enquadram nesse erro.

Quinta questão (23 acertaram a questão, sendo 12 da Engenharia Química, cinco da Engenharia Mecânica e seis da Engenharia Metalúrgica):

O átomo de hidrogênio é composto por um próton no núcleo e um elétron, que se move ao redor do núcleo. Na teoria quântica de estrutura atômica supõe-se que o elétron não se mova em uma órbita bem definida. Ao contrário, ele ocupa um estado conhecido como orbital, que pode ser pensado como uma "nuvem" de carga negativa rodeando o núcleo. No estado de energia mais baixa, chamado estado fundamental, ou orbital $1 \mathrm{~s}$, é suposto que o formato do orbital seja uma esfera com centro no núcleo. Essa esfera é escrita em termos da função densidade de probabilidade

$$
\mathrm{p}(\mathrm{x})=\frac{4}{\alpha^{\mathbb{8}}} \mathrm{x}^{2} e^{-2 x / \propto}, \mathrm{x} \geq 0 .
$$

Onde $\alpha\left(\equiv 5,59.10^{-11} \mathrm{~m}\right)$ é o raio de Bohr. A integral

$\int_{0}^{\mathrm{n}} \frac{4}{\alpha^{3}} \mathrm{~s}^{2} e^{-2 s / \propto} \mathrm{ds}$

Fornece a probabilidade do elétron ser encontrado dentro da esfera de raio x metros centrada no núcleo. Verifique que $\mathrm{p}(\mathrm{x})$ é uma função densidade. Isto é:

(i) $\mathrm{p}(\mathrm{x}) \geq 0$, qualquer $\mathrm{x}$ do domínio e 


\section{(ii) $\int_{0}^{\text {ver }} \mathrm{p}(\mathrm{x}) \mathrm{dx}=1$}

Principais erros observados: a) Má compreensão do enunciado ocasionando a não resolução do problema. Erro cometido por 16 discentes; b) Erro associado a integração sem considerar que há composição na função e uso da integração por partes. Erro de integração. 18 discentes cometeram; c) Integraram coerentemente mas não fizeram uso do fato da integral ser imprópria. Erro de compreensão do que seja uma integral imprópria. 27 discentes se enquadram nesse erro.

\section{Conclusões e Sugestões.}

Todas as provas aplicadas consistiam de texto base com intuito de fornecer informação aos discentes dos conteúdos que deveriam ser utilizados em determinada situação. Ou seja, ler e interpretar cada questão faz parte da avaliação. Mesmo usando as sugestões de McDonald (2003), não foi percebida uma grande diferença de notas nas observações em cada prova.

Em relação à mudança no conteúdo, isto é, antecipar assuntos como método dos trapézios para cálculo de áreas, se comparado com outras turmas, observou-se que as dificuldades de compreensão das fórmulas são equivalentes. Idem para aplicações das regras de 1'Hopital. Usam indiscriminadamente sem observar o quando usar.

Se os erros observados são parecidos, por qual motivo modificar a sequência didática? Este questionamento, apresentado no início deste trabalho, tem um aspecto positivo: as aplicações ou os motivos do desenvolver determinados conteúdos são argumentados de maneira participativa.

Fica, como consideração final, a necessidade de refazer a mudança didática com outras turmas de engenharias para ter mais subsídios para melhor analisar os erros. Não obstante, observar turmas de Cálculo Avançado, que é a disciplina seguinte ao Cálculo Fundamental, para tentar perceber se de fato ocorreu uma aprendizagem, independentemente da sequência seguida.

\section{Referências}

BARBOSA, Jane. A prática pedagógica no ensino superior. Rio de Janeiro: UCB/CEP, 2006.

BRASIL. MEC. Parâmetros Curriculares Nacionais. Temas Transversais. Brasília: MEC/SEF. 1998.

CURY, H. N. Análise de erros: o que podemos aprender com as respostas dos alunos. Belo Horizonte: Autêntica, 2007.

HOFFMAN, Jussara. Avaliação mediadora: uma prática em construção da pré-escola à universidade. Porto Alegre: Educação e realidade, 1994. 
LUCKESI, Cipriano C. Avaliação da aprendizagem escolar. 8. ed. São Paulo: Cortez, 1994.

McDONALD, B. C. (org.). Esboços em Avaliação Educacional. Fortaleza: Editora da UFC, 2003.

PERRENOUD, Philip. Avaliação. São Paulo: Artmed, 1999.

STEWART, J. Cálculo. 1v. - 6.ed. - São Paulo: Cengage, 2010.

THOMAS, G. Cálculo. 1v. - 11.ed. - São Paulo: Addison Wesley, 2009.

\section{Como citar este artigo (Formato ABNT):}

BRANDÃO, Jorge C.; Magalhães, Elisângela B.; COSTA, Emília da. Novas Estratégias e velhoe erros: Uma introdução ao cálculo para alunos de engenharias. Disponível em: http://www.idonlinerevistadepsicologia.emnuven.com.br/id >. Id on Line Revista de Psicologia, Novembro de 2015, vol.9, n.28. p. 30-41. ISSN 1981-1189.

Recebido: $10 / 05 / 2015$.

Aceito: 26/06/2015 